\title{
UMA DISCUSSÃO SOBRE MODELOS MECÂNICOS DE LARINGE PARA SÍNTESE DE VOGAIS
}

\author{
Edson Cataldo ${ }^{1}$ \\ Rubens Sampaio ${ }^{2}$ \\ Lucas Nicolato ${ }^{3}$
}

\begin{abstract}
Resumo: O mecanismo mais importante relacionado à produção de voz é o das cordas vocais. O ar proveniente dos pulmões é forçado através do pequeno espaço existente entre as cordas vocais, causando o movimento das cordas vocais em uma freqüência determinada pela tensão dos músculos associados. Este movimento causa a modificação do fluxo de ar, resultando em pulsos de ar que serão amplificados e modificados pelas cavidades oral e nasal até ser irradiado pela boca. Este complexo processo é modelado por um sistema de equações íntegro-diferenciais. Neste artigo, apresentamos três modelos mecânicos das cordas vocais e um deles, o mais complexo (modelo de Titze), é descrito completamente. Neste caso, discutimos todo o processo usado para a produção de vogais. Os resultados obtidos com os três modelos são comparados. Embora os resultados obtidos sejam preliminares, são satisfatórios na produção de vogais e dão razões para acreditar que podemos continuar nesse caminho para a síntese articulatória da voz.
\end{abstract}

Palavras-chave: Modelagem, simulação, produção de vogais, processamento de sinais.

Abstract: The most important mechanism involved in the production of the voice is played by the vocal cord. Air coming from the lungs are forced through the narrow space between the two sides of the vocal cord that are set in motion in a frequency that is governed by the tension in the attached muscles. The vocal cord change the type of flow that comes from the lungs and as the flow passes through the oral and nasal cavities it is amplified and changed until it is radiated from the mouth. This complex process can be modeled by a system of integral-differential equations. In this paper is presented three mechanical models of the vocal cord and the most complex one, the Titze model, is described completely. In this case, we discuss all of the process used for the production of vowels. The results of the three models are compared. Although the models are rather crude, the results obtained in the production of vowels are satisfactory and give reason to believe that we can follow this way to the articulatory synthesis of voice.

Keywords: Modeling, Simulation, Voice production, Signal Processing

\footnotetext{
${ }^{1}$ UFF, Departamento de Matemática Aplicada, PGMEC - Programa de pós-graduação em Engenharia Mecânica, Programa de pós-graduação em Engenharia de Telecomunicações. ecataldo@mec.puc-rio.br ${ }^{2}$ PUC-Rio- Departamento de Engenharia Mecânica - rsampaio@mec.puc-rio.br

${ }^{3}$ UFF -Engenharia de Telecomunicações - lucasnicolato@yahoo.com.br
} 


\section{INTRODUÇÃO}

Uma das principais motivações para o entendimento do mecanismo da produção de voz está no fato que a voz humana é um dos principais meios de comunicação.

A produção da voz se inicia com uma contração-expansão dos pulmões. Criase, assim, uma diferença entre a pressão do ar nos pulmões e a pressão do ar na frente da boca, causando um escoamento de ar. O escoamento passa pela laringe e, antes homogêneo, vai se transformando em uma série de pulsos (conhecidos como trem de pulsos ou sinal glotal) de ar que chegam na boca e na cavidade nasal. Os pulsos de ar são modulados pela língua, pelos dentes e lábios; isto é, pela geometria destes órgãos, de forma a produzir o que conhecemos por voz. O sinal glotal, porém, possui propriedades importantes de difícil reprodução que estão intimamente ligadas às características anatômicas e fisiológicas da laringe.

Atualmente, a teoria mais aceita para a descrição do sinal glotal (isto é, o aparecimento do trem de pulsos) é a teoria chamada de aerodinâmicamioelástica, proposta por van den Berg (1958) e Titze (1980). Esta teoria postulou que o movimento de abrir e fechar as cordas vocais é regido pelas propriedades mecânicas dos tecidos musculares que constituem, principalmente, as cordas vocais e pelas forças aerodinâmicas que se distribuem ao longo da laringe durante a fonação. A ação neural consiste apenas em aproximar as cordas vocais de tal forma que a superfície destas vibrem.
Para facilitar o estudo do sistema de produção da voz, normalmente o reduzimos a quatro grupos distintos, em relação à onda sonora que é produzida ou modificada pelos órgãos. O primeiro grupo, que chamaremos de grupo da respiração, corresponde à produção de um fluxo de ar; que inicia-se nos pulmões e termina no final da traquéia. Na faringe, encontram-se os órgãos do segundo grupo, responsáveis pela produção do sinal glotal, que chamaremos de grupo da vocalização. O sinal glotal é um sinal de baixa intensidade, que necessita ser amplificado e que determinadas componentes harmônicas sofram "ênfase", de maneira que os fonemas sejam caracterizados. Chamaremos este grupo de grupo de ressonância. Esse fenômeno ocorre na passagem do ar pelo chamado trato vocal (porção que vai da laringe até a boca). Finalmente, as ondas de pressão são irradiadas quando chegam à boca. A esse grupo chamaremos de grupo da irradiação.

Um dos principais diferenciadores do som é a forma de excitação. Identificamos dois tipos básicos de excitação: (1) vocálico e (2) nãovocálico. Neste trabalho, discutiremos apenas a produção de sons vocálicos, no nosso caso, a produção de vogais.

\section{MODELAGEM MATEMÁTICA}

Na produção de vogais, o fluxo de ar proveniente dos pulmões é interrompido pela vibração quaseperiódica das cordas vocais, conforme ilustrado na figura a seguir.

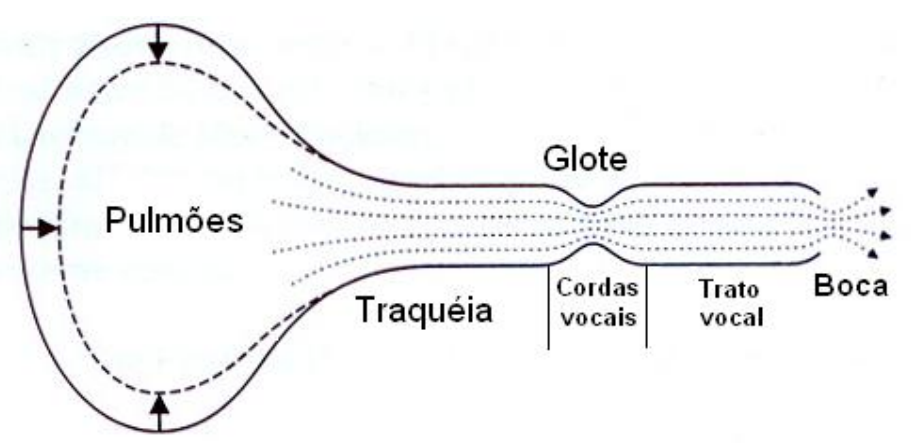

Fig.1 - Representação esquemática do sistema de produção da voz. 
A partir da representação esquemática do sistema de produção de voz, podemos observar os quatro grupos destacados anteriormente: respiração (pulmões e traquéia), vocalização (cordas vocais), ressonância (trato vocal) e radiação (boca).

Nas últimas décadas, a dinâmica das cordas vocais tem sido extensivamente estudada e alguns modelos mecânicos foram desenvolvidos. Esses modelos diferem pela representação que fazem das cordas vocais, vistas como sistemas mecânicos que modulam a passagem do ar.

Dentre esses modelos podemos destacar o modelo de uma única massa (Flanagan and Landgraf, 1968), modelos considerando duas massas (Ishizaka and Flanagan, 1972, 1977; Flanagan e Ishizaka, 1978; Koizumi et al, 1987), modelo considerando três massas (Titze, 1994), modelo com múltiplas massas (Titze, 1973, 1974), e modelos contínuos (Titze e Strong, 1975; Titze e Talkin, 1979; Titze, 1984). Há modelos bem mais sofisticados, como o apresentado por Rosa (2002).

Podemos considerar que a naturalidade da voz sintetizada está relacionada com o número de graus de liberdade do sistema considerado para representar a dinâmica das cordas vocais. É esperado, portanto, que os modelos de múltiplas massas e o modelo contínuo sejam superiores aos modelos de duas massas em termos da capacidade de produção da voz sintética. Porém, consideraremos neste artigo, apenas os modelos de uma, duas e três massas. A grande vantagem desses modelos é a facilidade de implementação. Discutiremos e compararemos os resultados obtidos com esses modelos.

Como a produção da voz é caracterizada pela mudança da forma do trato vocal, pode-se esperar que um modelo mais realista de trato vocal consistiria de um tubo que variasse como uma função do tempo e da posição ao longo do eixo de propagação do som. A formulação de um modelo desse tipo do trato vocal pode ser extremamente ENGEVISTA, v. 6, n. 1, p. 47-57, abr. 2004 complexa. Um método de simplificação deste modelo é o de representar o trato vocal como uma série de tubos acústicos concatenados. Devemos destacar que não é apenas a mudança da forma do trato vocal a responsável pela produção da voz, há também fatores, como a pressão dos pulmões, entre outros. No entanto, para a modelagem tratada aqui, tomamos a pressão dos pulmões constantes. O que pode ser considerada para o caso de modelagem de vogal sustentada.

Para o caso da produção de vogais, consideramos que a área da seção do tubo varia apenas com a posição e não com o tempo.

\section{APRESENTAÇÃO MODELOS}

\subsection{Introdução}

Nesta seção, apresentaremos os três modelos a serem usados para a produção de vogais. Para os dois primeiros modelos faremos uma breve discussão, sem considerar muitos detalhes. Porém, para o terceiro modelo, faremos uma descrição mais detalhada, pois é o modelo considerado mais completo dentre os estudados aqui. Servirá como base para a discussão dos resultados desejados.

\subsection{Modelo de Flanagan-Landgraf (1968)}

O primeiro modelo a ser discutido será o modelo de Flanagan e Landgraf (1968), cujo circuito acústico para representar o sistema vocal é o da Fig.2. Um circuito acústico é construído levando-se em consideração a analogia existente entre um circuito elétrico linear RLC (Resistência - Indutância Capacitância) em séria com um sistema mecânico linear Massa-MolaAmortecedor. Suas dinâmicas são dadas por equações diferenciais lineares de segunda ordem, onde o equivalente da Indutância é a Massa, da Capacitância é a Mola e da Resistência é o Amortecedor. 


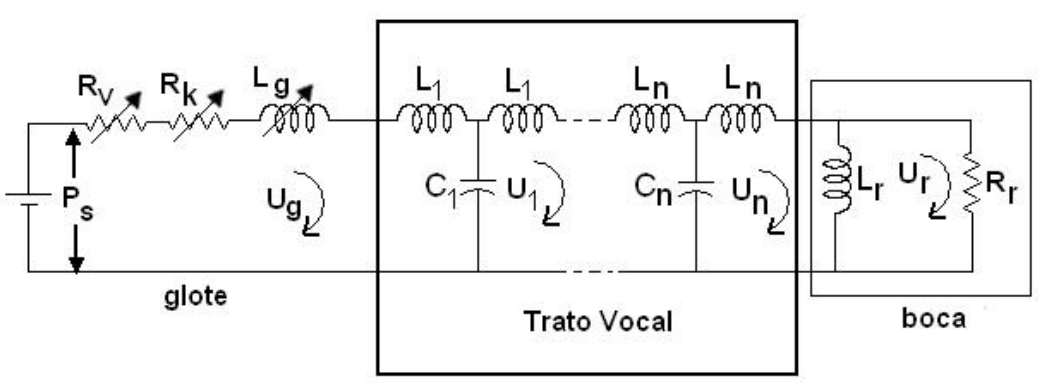

Fig.2 - Representação do circuito acústico para a produção de vogais.

No caso da produção de vogais, os pulmões aparecem como uma fonte de pressão constante e de baixa impedância. Além disso, a queda de pressão através das (grandes) áreas dos brônquios e da traquéia é relativamente pequena. Assim, aproximamos a pressão subglotal pela bateria esquematizada acima com valor de tensão $\mathrm{P}_{\mathrm{s}}$. Usando resultados experimentais obtidos por van den Berg (1957), a impedância glotal $\quad\left(\mathrm{R}_{\mathrm{v}}\right)$ dependente do tempo, a resistência cinética $\left(R_{k}\right)$ dependente do fluxo e a inertância $\left(\mathrm{L}_{\mathrm{g}}\right)$ devido à massa do pulso de ar glotal são dadas em termos da viscosidade cinemática do ar, da espessura das cordas vocais, dos comprimentos das cordas vocais, das áreas do orifício glotal, da densidade do ar e do fluxo do ar através do orifício glotal. Esses valores podem ser encontrados em Flanagan e Landgraf (1968).

Neste modelo, as cordas vocais são consideradas como um sistema massamola-amortecedor (massa $\mathrm{M}$, constante da mola $\mathrm{K}$ e amortecimento $\mathrm{B}$ ). O sistema é forçado por uma força $F(t)$, dada pelo produto da pressão do ar na glote pela área da superfície intraglotal. A força age na face da corda vocal, como descrito na figura a seguir. A força é distribuída e sua resultante, que não aparece na Fig. 3, pode ser pensada como aplicada na massa $\mathrm{M}$.

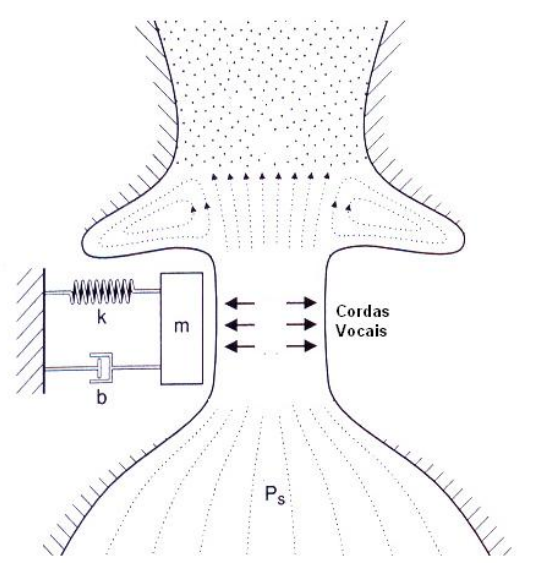

(b)

Fig.3 - (a) Modelo mecânico de segunda ordem para as cordas vocais. (b) Sistema vocal para o Modelo de Flanagan e Landgraf (1968).

\subsection{Modelo de Ishizaka e Flanagan (1972)}

Repetidas observações do movimento das cordas vocais, em câmera lenta, têm demonstrado que as cordas vocais não se movem como corpos rígidos. A mucosa vibra em um movimento que pode ser ENGEVISTA, v. 6, n. 1, p. 47-57, abr. 2004 pensado quase que independente do corpo que ela recobre. Devido a essas observações dois modelos podem ser criados, considerando-se o movimento de duas ou três massas.

Nesta seção, apresentaremos o modelo de duas massas e na próxima seção, o modelo com três massas. 
O sistema considerado para este modelo é mostrado na figura a seguir:

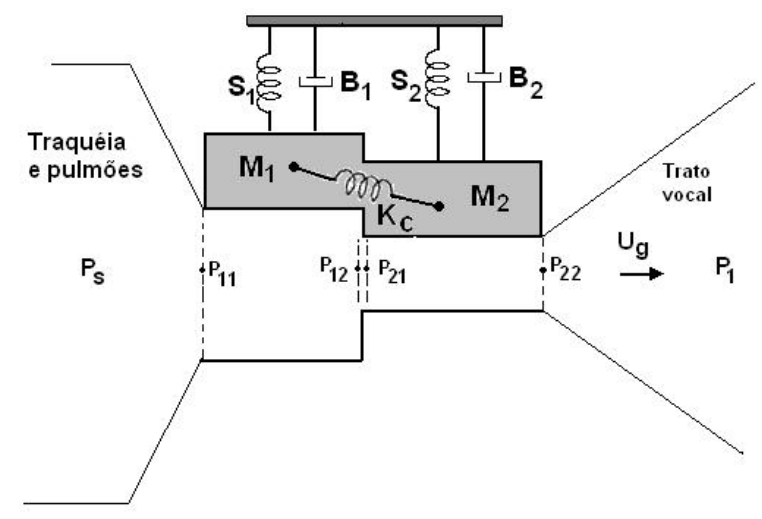

Fig.4 - Modelo mecânico de segunda ordem para as cordas vocais - modelo de Flanagan e Ishizaka (1972).

O circuito acústico utilizado para este modelo será o mesmo do modelo de Titze, discutido a seguir. Desta forma, deixaremos para apresentá-lo na discussão do referido modelo.

O sistema considerado para a dinâmica das cordas vocais é um sistema de dois graus de liberdade. As molas $\mathrm{S}_{1} \mathrm{e}$ $\mathrm{S}_{2}$ são não-lineares e a mola $\mathrm{K}_{\mathrm{C}}$ é linear.

\subsection{Modelo de Titze (1994)}

Este modelo considera a inclusão de uma terceira massa em relação ao modelo anterior, conforme descrito na Fig. 5:

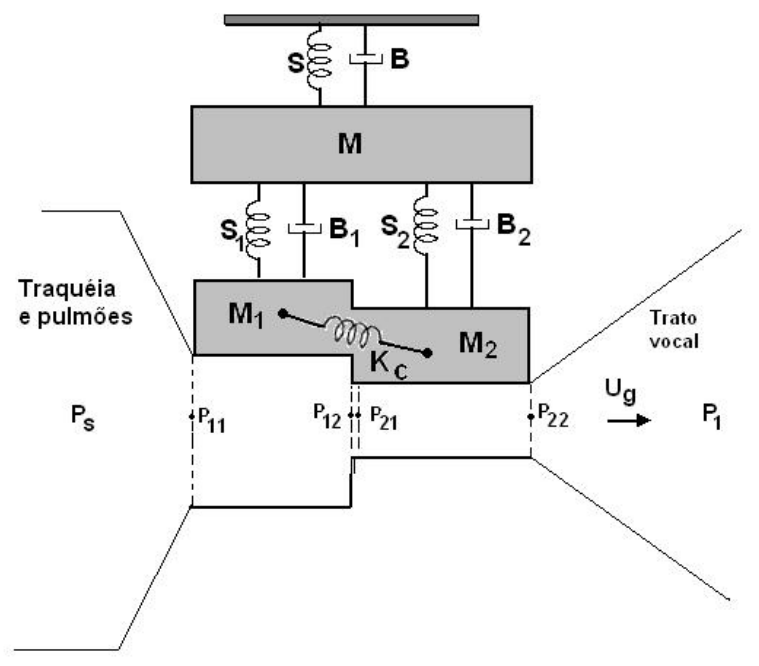

Fig.5 - Modelo das cordas vocais para o modelo de Titze (1994).

O sistema é composto por três massas ( $\mathrm{M}, \mathrm{M}_{1}$ e $\left.\mathrm{M}_{2}\right)$. A essas massas são permitidos apenas movimentos perpendiculares ao tubo vocal $\mathrm{x}, \mathrm{x}_{1} \mathrm{e}$ $\mathbf{x}_{2}$. As duas massas $\mathrm{M}_{1}$ e $\mathrm{M}_{2}$ são acopladas por uma mola linear $\mathrm{K}_{\mathrm{c}}$. As molas $S, S_{1}$ e $S_{2}$ são representações equivalentes da tensão nas cordas vocais e têm características não-lineares. A relação não-linear entre a deflexão a partir da posição de equilíbrio e a força requerida para produzir tal deflexão é dada por $\mathrm{f}=\mathrm{Kx}\left(1+\eta \mathrm{x}^{2}\right)$, no caso da mola $\mathrm{S}$, onde $\mathrm{f}$ é a força requerida para produzir $\mathrm{x}, \mathrm{K}$ é a rigidez linear e ๆé o 
coeficiente que descreve a nãolinearidade da mola $\mathrm{S}$. $\quad$ No caso das molas $\mathrm{S}_{1}$ e $\mathrm{S}_{2}$, Consideramos $f_{S_{j}}=K_{j} x_{j}\left(1+\eta_{k_{j}} x_{j}^{2}\right), j=1,2$, onde $f_{s j}$ é a força requerida para movimentar cada massa $M_{j}, K_{j}$ é a rigidez linear, e $\eta_{k_{j}}$ é o coeficiente que descreve a nãolinearidade da mola $S_{j}$, sendo positiva neste caso.

Durante o fechamento da glote, consideramos uma força de contato agindo quando as massas colidem. Essa força causará deformação nas cordas vocais. A força de restauração durante esse processo de colisão pode ser representada por uma mola equivalente $\mathrm{S}_{\mathrm{h}_{\mathrm{j}}}, \mathrm{j}=1,2$. Consideramos uma característica não-linear para essa mola equivalente $S_{h_{j}}$; isto é,

$$
f_{h_{j}}=h_{j}\left(x_{j}+\frac{A_{g 0 j}}{2 l_{g}}\right)\left\{1+\eta_{h_{j}}\left(x_{j}+\frac{A_{g 0 j}}{2 l_{g}}\right)^{2}\right\}
$$

para $\quad x_{j}+\frac{A_{g 0 j}}{2 l_{g}} \leq 0, j=1,2$

sendo $f_{h_{j}}$ a força requerida para produzir a deformação na massa, $M_{j}$, durante a colisão, $h_{j}$ é a rigidez linear e $\eta_{h_{j}}$ é um coeficiente positivo representando a nãolinearidade das cordas vocais em contato. A força resultante agindo em $\mathrm{M}_{\mathrm{j}}$ durante o fechamento é, então, a soma de $\mathrm{f}_{\mathrm{S}_{\mathrm{j}}}$ com $\mathrm{f}_{\mathrm{h}_{\mathrm{j}}} \cdot \mathrm{A}_{\mathrm{g} 0 \mathrm{j}}$ é a área da região existente entre as cordas vocais quando estão em repouso.

Tomando como base as relações entre as diferenças de pressões na glote, como descrito em Flanagan e Ishizaka (1972), a impedância acústica do orifício glotal constitui o circuito equivalente mostrado na figura a seguir:

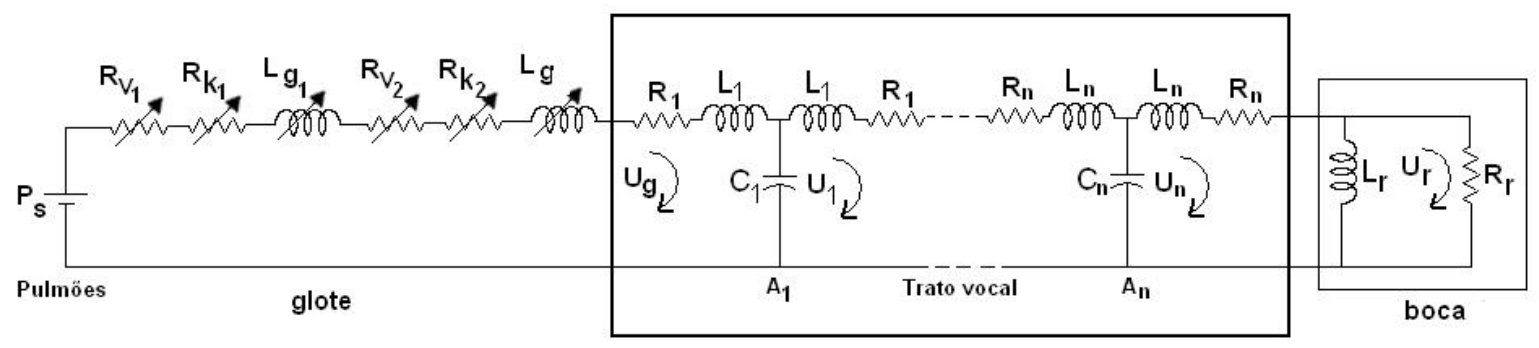

Fig. 6 - Circuito equivalente para o modelo de Flanagan e Ishizaka (1972) e de Titze (1994).

Sendo $R_{k 1}=\frac{0,19 \rho}{A_{g 1}^{2}}$,

$\mathrm{R}_{\mathrm{k} 2}=\frac{\rho\left[0,5-\frac{\mathrm{A}_{\mathrm{g} 2}}{\mathrm{~A}_{1}}\left(1-\frac{\mathrm{A}_{\mathrm{g} 2}}{\mathrm{~A}_{1}}\right)\right]}{\mathrm{A}_{\mathrm{g} 2}^{2}}$,

$\mathrm{R}_{\mathrm{v} 1}=12 \frac{\mu \mathrm{l}_{\mathrm{g}}^{2} \mathrm{~d}_{1}}{\mathrm{~A}_{\mathrm{g} 1}^{3}} \quad, \quad \mathrm{~L}_{\mathrm{g} 1}=\frac{\rho \mathrm{d}_{1}}{\mathrm{~A}_{\mathrm{g} 1}}$,

$\mathrm{R}_{\mathrm{v} 2}=12 \frac{\mu \mathrm{l}_{\mathrm{g}}^{2} \mathrm{~d}_{2}}{\mathrm{~A}_{\mathrm{g} 2}^{3}} \quad, \quad \mathrm{~L}_{\mathrm{g} 2}=\frac{\rho \mathrm{d}_{2}}{\mathrm{~A}_{\mathrm{g} 2}}$,
$\mathrm{A}_{\mathrm{g} 1}=\mathrm{A}_{\mathrm{g} 01}+2 \ell_{\mathrm{g}} \mathrm{x}_{1}$

$A_{\mathrm{g} 2}=A_{\mathrm{g} 02}+2 \ell_{\mathrm{g}} \mathrm{x}_{2}$.

Consideramos a viscosidade dinâmica do $\operatorname{ar}(\mu)$ igual a $1,84 \times 10^{-5}$, as espessuras $\mathrm{d}_{1}$ e $\mathrm{d}_{2}$ das cordas vocais iguais a 0,0032 $\mathrm{m}$, o comprimento $\ell_{\mathrm{g}}$ das cordas vocais igual a $1,8 \times 10^{-2} \mathrm{~m}$, as áreas neutras do orifício glotal $\mathrm{A}_{\mathrm{g} 01}, \mathrm{~A}_{\mathrm{g} 02}$ (cordas vocais em repouso) iguais a $0,05 \mathrm{~cm}^{2} \mathrm{e}$ a densidade do ar $(\rho)$ igual a $1,3 \mathrm{~g} / \mathrm{cm}^{3}$. 
Os deslocamentos das massas, $\mathrm{x}_{1} \mathrm{e}$ $\mathrm{x}_{2}$, são obtidos pelas equações a seguir, que descrevem a dinâmica das cordas vocais para este modelo.

$$
\left\{\begin{array}{l}
M \ddot{x}+B \dot{x}+S(x)+S_{1}\left(x-x_{1}\right)+B_{1}\left(\dot{x}-\dot{x}_{1}\right)+S_{2}\left(x-x_{2}\right)+B_{2}\left(\dot{x}-\dot{x}_{2}\right)=0 \\
M_{1} \ddot{x}_{1}+S_{1}\left(x_{1}-x\right)+B_{1}\left(\dot{x}_{1}-\dot{x}\right)+k_{c}\left(x_{1}-x_{2}\right)=F_{1} \\
M_{2} \ddot{x}_{2}+S_{2}\left(x_{2}-x\right)+B_{2}\left(\dot{x}_{2}-\dot{x}\right)+k_{c}\left(x_{2}-x_{1}\right)=F_{2}
\end{array}\right.
$$

As molas $\mathrm{S}, \mathrm{S}_{1} \mathrm{eS}_{2}$ seguem as características não-lineares descritas anteriormente. As forças $\mathrm{F}_{1}$ e $\mathrm{F}_{2}$ são calculadas em termos das pressões do ar na glote (pressão $\mathrm{x}$ área). O trato vocal é representado por uma linha de transmissão de $\mathrm{n}$ cilindros, de paredes rígidas, áreas das seções transversais $A_{1}, A_{2}, \ldots, A_{n}$, e os comprimentos dos cilindros $\ell_{1}, \ell_{2}, \ldots, \ell_{\mathrm{n}}$. Os valores das indutâncias são dadas por $L_{j}=\frac{\rho \ell_{j}}{2 A_{j}}$, $\mathrm{j}=1, \ldots, \mathrm{n}$ e as capacitâncias são dadas por $\mathrm{C}_{\mathrm{j}}=\frac{\ell_{\mathrm{j}} \mathrm{A}_{\mathrm{j}}}{\rho \mathrm{c}^{2}}, \mathrm{j}=1, \ldots, \mathrm{n}$, onde cé a velocidade do som. As resistências são calculadas pela fórmula
$R_{j}=\frac{s_{j}}{A_{j}^{2}} \sqrt{\rho \mu \omega / 2}$, sendo $s_{j}$ o perímetro da circunferência da j-ésima seção e $\omega$ é a freqüência em radianos por segundo. A linha de transmissão termina com uma carga de radiação representada pela impedância $Z_{\mathrm{r}}$ (formada por uma resistência $R_{r}=\left(\frac{128 \rho c}{9 \pi^{2} A_{n}}\right)$ e por uma indutância $L_{r}=\frac{8 \rho}{3 \pi} \sqrt{\pi A_{n}}$ colocadas em paralelo).

Usando leis das malhas, chegamos às seguintes equações para o circuito da figura:

Malha 0: correspondente à seção das cordas vocais

$$
\left(\mathrm{R}_{\mathrm{k} 1}+\mathrm{R}_{\mathrm{k} 2}\right)\left|\mathrm{U}_{\mathrm{g}}\right| \mathrm{U}_{\mathrm{g}}+\left(\mathrm{R}_{\mathrm{v} 1}+\mathrm{R}_{\mathrm{v} 2}\right) \mathrm{U}_{\mathrm{g}}+\left(\mathrm{L}_{\mathrm{g} 1}+\mathrm{L}_{\mathrm{g} 2}\right) \frac{\mathrm{dU}_{\mathrm{g}}}{\mathrm{dt}}+\mathrm{L}_{1} \frac{\mathrm{dU} \mathrm{g}}{\mathrm{dt}}+\mathrm{R}_{1} \mathrm{U}_{\mathrm{g}}+\frac{1}{\mathrm{C}_{1}} \int_{0}^{\mathrm{t}}\left(\mathrm{U}_{\mathrm{g}}-\mathrm{U}_{1}\right) \mathrm{dt}-\mathrm{P}_{\mathrm{s}}=0
$$

Malha 1: correspondente à primeira seção do trato vocal

$$
\left(\mathrm{L}_{1}+\mathrm{L}_{2}\right) \frac{\mathrm{dU}}{\mathrm{dt}}+\left(\mathrm{R}_{1}+\mathrm{R}_{2}\right) \mathrm{U}_{1}+\frac{1}{\mathrm{C}_{2}} \int_{0}^{\mathrm{t}}\left(\mathrm{U}_{1}-\mathrm{U}_{2}\right) \mathrm{dt}+\frac{1}{\mathrm{C}_{1}} \int_{0}^{\mathrm{t}}\left(\mathrm{U}_{1}-\mathrm{U}_{\mathrm{g}}\right) \mathrm{dt}=0
$$

Malha 2: correspondente à segunda seção do trato vocal

$$
\left(\mathrm{L}_{2}+\mathrm{L}_{3}\right) \frac{\mathrm{dU}_{2}}{\mathrm{dt}}+\left(\mathrm{R}_{2}+\mathrm{R}_{3}\right) \mathrm{U}_{2}+\frac{1}{\mathrm{C}_{3}} \int_{0}^{\mathrm{t}}\left(\mathrm{U}_{2}-\mathrm{U}_{3}\right) \mathrm{dt}+\frac{1}{\mathrm{C}_{2}} \int_{0}^{\mathrm{t}}\left(\mathrm{U}_{2}-\mathrm{U}_{1}\right) \mathrm{dt}=0
$$

e, assim por diante, até a penúltima malha.

Malha n: correspondente à última seção do trato vocal - n-ésima seção

$$
\left(\mathrm{L}_{\mathrm{n}}+\mathrm{L}_{\mathrm{R}}\right) \frac{\mathrm{d} \mathrm{U}_{\mathrm{n}}}{\mathrm{dt}}+\mathrm{R}_{\mathrm{n}} \mathrm{U}_{\mathrm{n}}-\mathrm{L}_{\mathrm{R}} \frac{\mathrm{dU}_{\mathrm{R}}}{\mathrm{dt}}+\frac{1}{\mathrm{C}_{\mathrm{n}}} \int_{0}^{\mathrm{t}}\left(\mathrm{U}_{\mathrm{n}}-\mathrm{U}_{\mathrm{n}-1}\right) \mathrm{dt}=0
$$


Malha da irradiação na boca: $L_{R} \frac{d\left(U_{R}-U_{n}\right)}{d t}+R_{R} U_{R}=0$

Para resolver o sistema obtido, usamos equações de diferença, considerando:

$$
\frac{d f}{d t} \cong \frac{f\left(t_{i}\right)-f\left(t_{i-1}\right)}{t_{i}-t_{i-1}} \quad \text { e } \int_{a}^{b} f(t) d t \cong T \sum_{j=0}^{i-1} f\left(t_{j}\right) \text {, sendo } T=\frac{b-a}{i}
$$

conforme proposto por Flanagan e Landgraf (1972).

Comparando os modelos, verificou-se que o terceiro requeria mais tempo de CPU, mas, em compensação, apresentou os melhores resultados, principalmente no que diz respeito ao som produzido. Como resultado, podemos obter a função que descreve a área da seção glotal Ag (observamos que esse valor depende do trato vocal), a função que descreve o fluxo glotal Ug, a pressão acústica na boca (representada pelo produto da corrente $U_{n}$ pela impedância de radiação) e a função resposta em freqüência do trato vocal. Um outro resultado, igualmente importante, é o som obtido a partir da modelagem. Para obtenção dos resultados descritos acima, consideramos tratos vocais de comprimento $17 \mathrm{~cm}$. Faremos a comparação considerando a produção da vogal 'a'. Os resultados obtidos foram comparados com os encontrados nos trabalhos de Flanagan e Landgraf (1968) e de Ishizaka e Flangan (1972). O modelo chamado de Modelo de

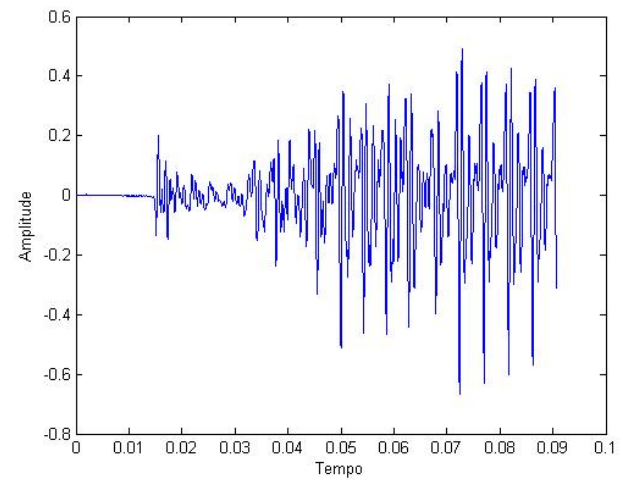

$\begin{array}{cc}\text { (a) } & \text { (b) } \\ \text { Fig. } 7 \text { - Sinal de voz correspondente à vogal 'a' (sustentada): (a) sinal durante todo o }\end{array}$

Titze está apenas comentado em Titze (1994), fizemos, assim, a sua implementação.

\section{COMPARAÇÃO ENTRE OS GRÁFICOS DA ÁREA DA SEÇÃO GLOTAL (Ag), DO FLUXO GLOTAL (Ug) e DA PRESSÃO ACÚSTICA NA BOCA}

Faremos a comparação entre os gráficos da área glotal (Ag), do fluxo glotal (Ug) e da pressão acústica na boca, para os três modelos considerados. Mostramos, primeiro, na Fig. 7, o sinal obtido de um sinal de voz (real) na emissão de uma vogal 'a' sustentada.

Mostramos, na Fig. 8, os gráficos relacionados à produção da vogal 'a', usando os três modelos discutidos. Usamos dezessete seções do trato vocal, com valores sugeridos por Koizumi et al (1987). tempo da emissão da voz, (b) apenas um trecho do sinal-destaque para a periodicidade do sinal. 
MODELO de 1968

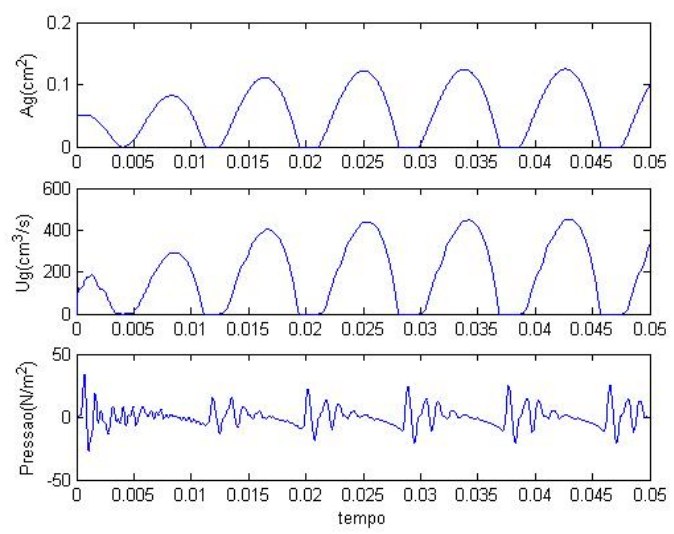

MODELO de 1972
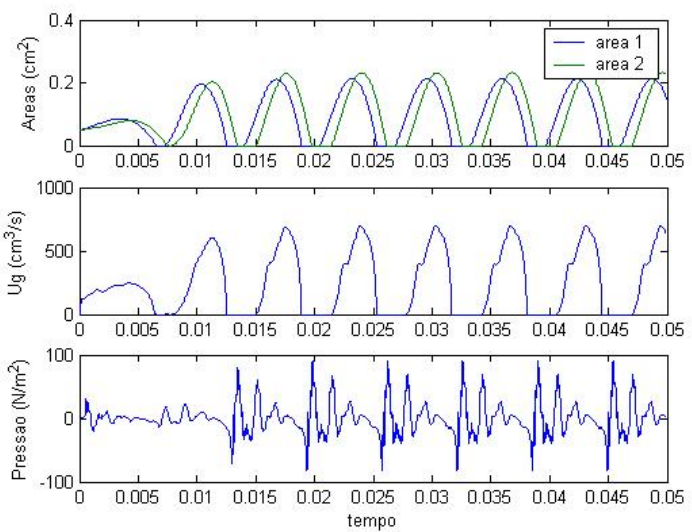

MODELO de TITZE
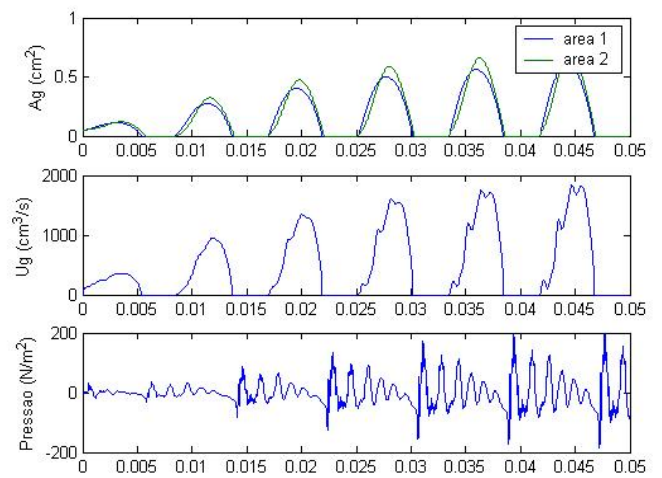

Fig. 8- Gráficos da(s) área(s) da seção glotal, do fluxo glotal e da pressão irradiada para os três modelos considerados, no caso da emissão da vogal ‘a' (vogal 'a’ sustentada).

5. EXEMPLO DE FUNÇÃO RESPOSTA EM FREQÜÊNCIA OBTIDA

Mostramos na Fig. 9 o gráfico de amplitudes da função resposta em freqüência obtida através da razão entre o espectro de amplitude da corrente $\mathrm{U}_{\mathrm{m}}$ do circuito acústico e o espectro de

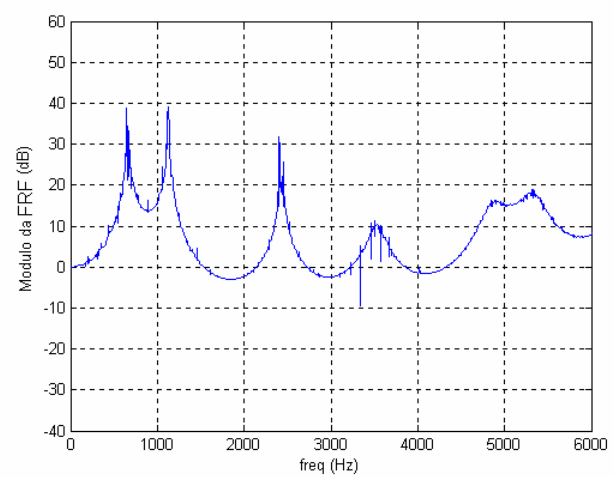

(a) amplitude da corrente de entrada $U_{g}$, para os três modelos tratados aqui. Os valores típicos para os quatro primeiros formantes para a vogal 'a', de acordo com Rabiner e Schafer (1978) são: 650 $\mathrm{Hz}, 1075 \mathrm{~Hz}, 2463 \mathrm{~Hz}$ e $3558 \mathrm{~Hz}$. Podemos comparar esses valores com os verificados nos gráficos da Fig. 9.

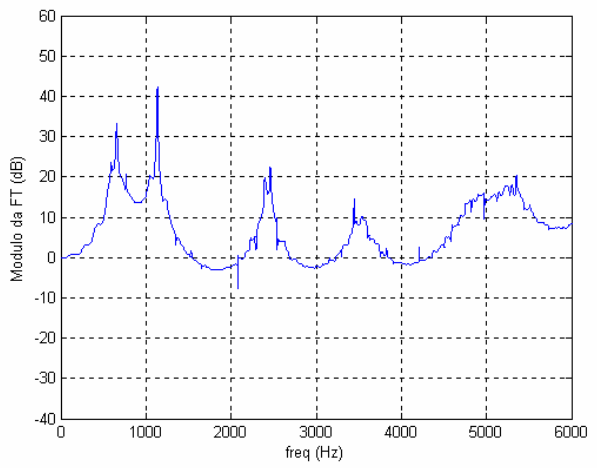

(b)

Fig. 9 - Gráfico de amplitudes da função resposta em freqüência, na síntese da vogal 'a' considerando os modelos: (a) Modelo de 1968, (b) Modelo de 1972 


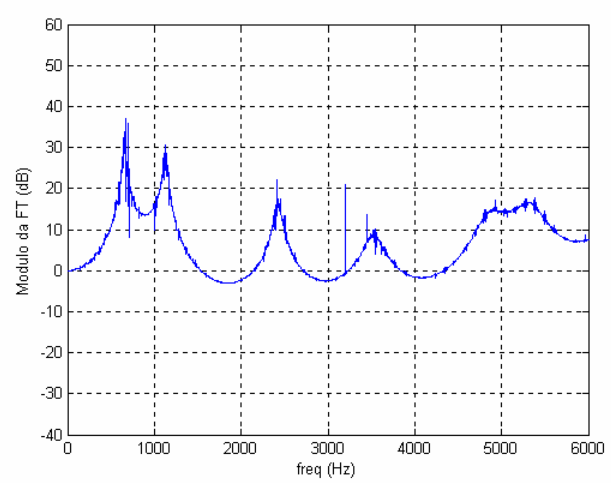

Fig. 10 - Gráfico de amplitudes da função resposta em freqüência, na síntese da vogal 'a' considerando o Modelo de Titze.

\section{CONCLUSÕES}

Ao compararmos os três modelos discutidos para a produção de vogais, observamos que os gráficos no domínio do tempo são bem parecidos com os gráficos obtidos na produção da vogal 'a' real. Podemos observar os gráficos do fluxo glotal, da área glotal e da pressão acústica dos três modelos e verificar que, no terceiro modelo, com o acréscimo da terceira massa, há uma demora para a estabilização dos pulsos, porém os sinais, em cada período, são mais próximos dos obtidos com um sinal real. Quanto ao som produzido, conseguimos identificar uma sensível melhora, em relação ao obtido com o segundo modelo e uma melhora substancial com 0 som produzido pelo primeiro modelo. A melhora relatada aqui está apenas na sensação de um som mais natural identificado pelo ouvido humano. Através das funções resposta em freqüência obtidas para os três modelos, na síntese da vogal 'a', podemos determinar seus formantes e compara-los com valores reais por Rabiner e Schafer (1978), observando que são valores muito próximos.

\section{REFERÊNCIAS}

Flanagan, J. e Landgraf, L., 1968, “Selfoscillating source for vocal-tract synthesizers",

IEEE Trans. On Audio and Eletroacoustics, Vol. 16, pp. 57-64.
Ishizaka, K. and Flanagan, J., 1972, "Synthesis of voiced sounds from two-mass model of the vocal cords", Bell Syst. Tech. Journal, Vol. 51, pp. 1233-1268.

Ishizaka, K. and Flanagan, J., 1977, "Acoustic properties of longitudinal displacement in

vocal cord vibration”, Bell Syst. Tech. Journal, Vol. 56, pp. 889-918.

Ishizaka, K. and Flanagan, J., 1978, "Computer model to characterize the air volume

displaced by the vibrating vocal cords", Journal of the Accoustical Society American,Vol. 63, pp. 15591565.

Koizumi, T.; Taniguchi, S.; Hiromitsu, S., 1987, "Two-mass models of the vocal cords for

natural sounding voice synthesis”, Journal of the Accoustical Society American, Vol. 82(4), pp. 1179-1192.

Rabiner, L.R. e Schafer, R.W., 1978, "Digital Processing of Speech Signals”, Prentice Hall, Englewood Cliffs, New Jersey.

Rosa, M. O., 2002, "Laringe Digital", Tese de doutorado, Universidade Federal de São

Carlos.

Titze, I. R., 1994, "Principles of Voice Production”, Prentice Hall, Englewood Cliffs, New Jersey.

Titze, I. R., 1973, “The Human Vocal Cords: A Mathematical Model-Part 
129-170.

Titze, I. R., 1974, "The Human Vocal Cords: A Mathematical Model-Part II”, Phonetica Vol. 29, pp. 1-21.

Titze, I. R., 1975, "Normal modes in vocal cord tissues", Journal of the Accoustical Society American, Vol. 57(3), pp. 736-744.

Titze, I. R., 1979, “A Theoretical study of the effects of various laryngeal configurations on the acoustics of phonation", Journal of the Accoustical Society American, Vol. 66(1), pp. 60-74.

Titze, I. R., 1980, "Comments on the myoelastic-aerodynamic theory of phonation", The Journal of the Acoustical Society of America, Vol. 23, pp. 495-510.

Titze, I. R., 1984, "Parametrization of the glottal area, glottal flow, and vocal fold contact area", Journal of the Accoustical Society American, Vol. 75, pp. 570-580.

Van den Berg, J. W., Zantema, J. T. e Doornenbal, P. Jr., 1957, “On the air resistance and the Bernoulli effect of the human larynx, Journal of Accoustical Society American, Vol. 29, pp. 626-631.

Van den Berg, J.,1958, "Myoelasticaerodynamic theory of voice production”, Journal of Speech and Hearing Research, Vol.1, pp. 227244. 